\title{
A System Matrix Equations over an Arbitrary Skew Field
}

\author{
Youcai Xue \\ School of Science \\ Zhejiang University of Science and Technology \\ 318, Liuhe Rod, Hangzhou 310023, China \\ Tel: 86-571-8507-0705_E-mail: xue_jian2008@sina.com
}

\begin{abstract}
In this paper, we give a practical solving method and an expression of general solutions of a system matrix equations $A_{1} X B_{1}=C_{1}$ and $A_{2} X B_{2}=C_{2}$ over an arbitrary skew field by using some matrix techniques and elementary operations on matrices.
\end{abstract}

Keywords: skew field, System matrix equations, Elementary operations on matrices

\section{Introduction}

It is well known that matrix equation is one of important contents of matrix study. In [1] an expression of general solutions of the matrix equation $A X B=C Y D$ over an arbitrary skew field was given. In this paper we consider the mentioned system matrix equations $A_{1} X B_{1}=C_{1} a n d A_{2} X B_{2}=C_{2}$ over an arbitrary skew field and give an expression of general solutions and a practical solving method of the matrix equation by using some matrix techniques and elementary operations on matrices with entries from an arbitrary skew field.

Throughout this paper we denote an arbitrary skew field by $F$, the set of all $m \times n$ matrixes over $F$ by $F^{m \times n}$, the set of all matrices in $F^{m \times n}$ with rank $r$ by $F_{r}^{m \times n}$, a $m \times m$ identity matrix by $I_{m}$, the rank of matrix $A$ by $\operatorname{rank} A$.

Now we introduce the following known lemmas.

Lemma $1.1^{[2]}$ Let $A \in F^{m \times n}$. Then $\operatorname{rank} A=r$ if and only if there exist $P \in F_{m}^{m \times m}$ and $Q \in F_{n}^{n \times n}$ such that $P A Q=\left[\begin{array}{cc}I_{r} & 0 \\ 0 & 0\end{array}\right]$

Lemma $1.2^{[3]}$ Suppose $P$ and $Q$ be invertible matrices over $F$. If the multiplication of matrices can be performed, then rank $A=\operatorname{rank} P A=\operatorname{rank} A Q=\operatorname{rank} P A Q$

for any matrix $A$ with entries from $F$.

Lemma $1.3^{[4]}$ Let $A \in F_{r}^{r \times r}$. Then

$\operatorname{rank}\left[\begin{array}{cc}A & B \\ C & D\end{array}\right]=r+\operatorname{rank}\left(D-C A^{-1} B\right)$

Lemma $1.4^{[5]}$ Let $A \in F^{n \times n}$. Then the following conditions are equivalent:

(i) $A$ is invertible;

(ii) $A$ is a product of elementary matrices;

(iii) $\operatorname{rank} A=n$

Lemma $1.5^{[5]}$ Let $A \in F^{m \times n}, E_{m}$ (resp. $E_{n}$ ) be the elementary matrix obtained by performing an elementary row[resp. column] operation $\mathrm{T}$ on $I_{m}$ (resp. $I_{n}$ ). Then $E_{m} A$ (resp. $A E_{n}$ ) is the matrix obtained by performing the operation $\mathrm{T}$ on $A$.

\section{Main Results}

Now we consider the matrix equation 


$$
\left\{\begin{array}{l}
A_{1} X B_{1}=C_{1} \\
A_{2} X B_{2}=C_{2}
\end{array}\right.
$$

where $A_{1} \in F_{r}^{m \times n}, A_{2} \in F_{t}^{p \times n}, B_{1} \in F_{s}^{l \times k}, B_{2} \in F_{w}^{l \times q}, C_{1} \in F^{m \times k}, C_{2} \in F^{p \times q}$.

(1) is equivalent to the following systems matrix equations

$$
\left\{\begin{aligned}
P A_{1} Q Q^{-1} X V_{0}^{-1} V_{0} B_{1} Q_{0} & =P C_{1} Q_{0} \\
V A_{2} Q Q^{-1} X V_{0}^{-1} V_{0} B_{2} U & =V C_{2} U
\end{aligned}\right.
$$

Where $P \in F_{m}^{m \times m}, Q \in F_{n}^{n \times n}, Q_{0} \in F_{k}^{k \times k}, V \in F_{p}^{p \times p}, U \in F_{q}^{q \times q}, V_{0} \in F_{l}^{l \times l}$.

Theorem $2.1 \quad$ For matrices $A_{1}, A_{2}, B_{1}, B_{2}$ in $P \in F_{m}^{m \times m}, Q \in F_{n}^{n \times n}, Q_{0} \in F_{k}^{k \times k}, V \in F_{p}^{p \times p}, U \in F_{q}^{q \times q}, V_{0} \in F_{l}^{l \times l}$, such that

(i) $P A_{1} Q=\left[\begin{array}{cc}I_{r} & 0 \\ 0 & 0\end{array}\right]_{m-r}{ }^{r},{ }^{\prime} V A_{2} Q=\left[\begin{array}{cccc}0 & 0 & I_{r_{2}} & 0 \\ I_{r_{1}} & 0 & 0 & 0 \\ 0 & 0 & 0 & 0\end{array}\right] \begin{gathered}r_{2} \\ r_{1} \\ p-r_{1}-r_{2}\end{gathered}$;

(ii) $V_{0} B_{1} Q_{0}=\left[\begin{array}{cc}I_{s} & 0 \\ 0 & 0\end{array}\right] l-s,{ }^{s} V_{0} B_{2} U=\left[\begin{array}{ccc}0 & I_{s_{2}} & 0 \\ 0 & 0 & 0 \\ I_{s_{1}} & 0 & 0 \\ 0 & 0 & 0\end{array}\right] \begin{gathered}s_{2} \\ s-s_{2} \\ s_{1} \\ l-s-s_{1}\end{gathered}$

Where $r_{1}+r_{2}=t, s_{1}+s_{2}=w$.

Proof We proof (ii).It follows from Lemma1.1 that there exist $V_{1} \in F_{l}^{I \times l}, Q_{1} \in F_{k}^{k \times k}$ such that $V_{1} B_{1} Q_{1}=\left[\begin{array}{cc}I_{s} & 0 \\ 0 & 0\end{array}\right] l-s$

Let $V_{1} B_{2}=\left[\begin{array}{l}W_{1} \\ W_{2}\end{array}\right]$,where $W_{1} \in F^{s \times q}, W_{2} \in F^{(l-s) \times q}$. For $W_{2}$, there exist $V_{2} \in F_{l-s}^{(l-s) \times(l-s)}, Q_{2} \in F_{q}^{q \times q}$,such that

$V_{2} W_{2} Q_{2}=\left[\begin{array}{rr}I_{s_{1}} & 0 \\ 0 & 0\end{array}\right] l-s-s_{1}$

Let $W_{1} Q_{2}=\left[\begin{array}{ll}W_{11} & W_{12}\end{array}\right]$, where $W_{11} \in F^{s \times s_{1}}, W_{12} \in F^{s \times\left(q-s_{1}\right)}$. It follows from Lemma 1.2 and 1.3 that

$\operatorname{rank} B_{2}=\operatorname{rank}\left[\begin{array}{cc}I_{s} & 0 \\ 0 & V_{2}\end{array}\right] V_{1} B_{2} Q_{2}=\operatorname{rank}\left[\begin{array}{cc}I_{s} & 0 \\ 0 & V_{2}\end{array}\right]\left[\begin{array}{c}W_{1} Q_{2} \\ W_{2} Q_{2}\end{array}\right]=\operatorname{rank}\left[\begin{array}{c}W_{1} Q_{2} \\ V_{2} W_{2} Q_{2}\end{array}\right]$

$=\operatorname{rank}\left[\begin{array}{cc}W_{11} & W_{12} \\ I_{s_{1}} & 0 \\ 0 & 0\end{array}\right]=s_{1}+\operatorname{rank} W_{12}$

Hence $\operatorname{rank} W_{12}=S-S_{1} \equiv S_{2}$

For $W_{12}$ there exist $V_{3} \in F_{s}^{s \times s}$ and $Q_{3} \in F_{q-s_{1}}^{\left(q-s_{1}\right) \times\left(q-s_{1}\right)}$ such that

$V_{3} W_{12} Q_{3}=\left[\begin{array}{cc}I_{s_{2}} & 0 \\ 0 & 0\end{array}\right] s-s_{2}$

Let

$$
V_{0}=\left[\begin{array}{cc}
V_{3} & \left(-V_{3} W_{11}, 0\right) V_{2} \\
0 & V_{2}
\end{array}\right] V_{1}, \quad Q_{0}=Q_{1}\left[\begin{array}{cc}
V_{3}^{-1} & 0 \\
0 & I_{k-s}
\end{array}\right], \quad U=Q_{2}\left(\begin{array}{cc}
I_{s_{1}} & 0 \\
0 & Q_{3}
\end{array}\right)
$$

by (3) (5)

$$
V_{0} B_{1} Q_{0}=\left[\begin{array}{cc}
V_{3} & \left(-V_{3} W_{11}, 0\right) V_{2} \\
0 & V_{2}
\end{array}\right] V_{1} B_{1} Q_{1}\left[\begin{array}{cc}
V_{3}^{-1} & 0 \\
0 & I_{k-s}
\end{array}\right]=\left[\begin{array}{cc}
I_{s} & 0 \\
0 & 0
\end{array}\right]
$$




$$
\begin{aligned}
& V_{0} B_{2} U=\left[\begin{array}{cc}
V_{3} & \left(-V_{3} W_{11}, 0\right) V_{2} \\
0 & V_{2}
\end{array}\right] V_{1} B_{2} Q_{2}\left[\begin{array}{cc}
I_{s_{1}} & 0 \\
0 & Q_{3}
\end{array}\right]=\left[\begin{array}{c}
V_{3} W_{1} Q_{2}+\left(-V_{3} W_{11}, 0\right) V_{2} W_{2} Q_{2} \\
V_{2} W_{2} Q_{2}
\end{array}\right]\left[\begin{array}{cc}
I_{s_{1}} & 0 \\
0 & Q_{3}
\end{array}\right] \\
= & {\left[\begin{array}{cc}
0 & V_{3} W_{12} \\
{\left[\begin{array}{ccc}
I_{s_{1}} \\
0
\end{array}\right]} & 0
\end{array}\right]\left[\begin{array}{cc}
I_{s_{1}} & 0 \\
0 & Q_{3}
\end{array}\right]=\left[\begin{array}{cc}
0 & V_{3} W_{12} Q_{3} \\
{\left[\begin{array}{ccc}
I_{s_{1}} \\
0
\end{array}\right]} & 0
\end{array}\right]=\left[\begin{array}{ccc}
0 & I_{s_{2}} & 0 \\
0 & 0 & 0 \\
I_{s_{1}} & 0 & 0 \\
0 & 0 & 0
\end{array}\right] }
\end{aligned}
$$

Similarly,(i) may be shown.

Let

(iii)

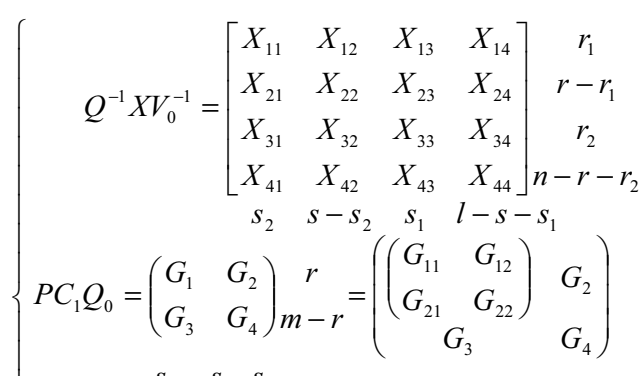

$$
\begin{aligned}
& V C_{2} U=\left(\begin{array}{ccc}
s_{2} & s-s_{2} & \\
E_{11} & E_{12} & E_{13} \\
E_{21} & E_{22} & E_{23} \\
E_{31} & E_{32} & E_{33} \\
s_{1} & s_{2} & k-s
\end{array}\right) r_{1}
\end{aligned}
$$

Theorem 2.2 Let $P, U, Q, Q_{0}, V, V_{0}, E_{i j}(i, j=1,2,3), G_{i j}(i, j=1,2), G_{k}(=1,2,3,4)$ be matrices mentioned in theorem 2.1 and (iii). Then (1) has solution if and only if $X_{i j}=G_{i j}(i, j=1,2), X_{33}=E_{11}, X_{31}=E_{12}, X_{13}=E_{21}, X_{11}=E_{22}=G_{11}, G_{i}=0(i=2,3,4) E_{j 3}=E_{3 j}=0(j=1,2,3) \quad$ Whence the general solution of (1) is

$$
X=Q\left[\begin{array}{llll}
G_{11} & G_{12} & E_{21} & X_{14} \\
G_{21} & G_{22} & X_{23} & X_{24} \\
E_{12} & X_{32} & E_{11} & X_{34} \\
X_{41} & X_{42} & X_{43} & X_{44}
\end{array}\right] V_{0}
$$

where,

$X_{14} \in F^{r_{1} \times\left(l-s-s_{1}\right)}, X_{23} \in F^{\left(r-r_{1}\right) \times s_{1}}, X_{24} \in F^{\left(r-r_{1}\right) \times\left(l-s-s_{1}\right)}, X_{32} \in F^{r_{2} \times\left(s-s_{2}\right)}$,

$X_{34} \in F^{r_{2} \times\left(l-s-s_{1}\right)}, X_{41} \in F^{\left(n-r-r_{2}\right) \times s_{2}}, X_{42} \in F^{\left(n-r-r_{2}\right) \times\left(s-s_{2}\right)}, X_{43} \in F^{\left(n-r-r_{2}\right) \times s_{1}}$,

$X_{44} \in F^{\left(n-r-r_{2}\right) \times\left(l-s-s_{1}\right)}$

are all any matrices over $F \quad$ with corresponding orders.

Proof For (1),i.e. for (2), by (iii),(2)is equivalent to the following systems matrix equations

$$
\left\{\begin{array}{l}
\left(\begin{array}{ll}
I_{r} & 0 \\
0 & 0
\end{array}\right)\left(\begin{array}{llll}
X_{11} & X_{12} & X_{13} & X_{14} \\
X_{21} & X_{21} & X_{21} & X_{21} \\
X_{31} & X_{32} & X_{33} & X_{34} \\
X_{41} & X_{42} & X_{43} & X_{44}
\end{array}\right)\left(\begin{array}{ll}
I_{s} & 0 \\
0 & 0
\end{array}\right)=\left(\left(\begin{array}{cc}
G_{11} & G_{12} \\
G_{21} & G_{22} \\
G_{3} & G_{2} \\
G_{4}
\end{array}\right)\right. \\
\left(\begin{array}{llll}
0 & 0 & I_{r_{2}} & 0 \\
I_{r_{1}} & 0 & 0 & 0 \\
0 & 0 & 0 & 0
\end{array}\right)\left(\begin{array}{llll}
X_{11} & X_{12} & X_{13} & X_{14} \\
X_{21} & X_{21} & X_{21} & X_{21} \\
X_{31} & X_{32} & X_{33} & X_{34} \\
X_{41} & X_{42} & X_{43} & X_{44}
\end{array}\right)\left(\begin{array}{ccc}
0 & I_{s 2} & 0 \\
0 & 0 & 0 \\
I_{s 1} & 0 & 0 \\
0 & 0 & 0
\end{array}\right)=\left(\begin{array}{lll}
E_{11} & E_{12} & E_{13} \\
E_{21} & E_{22} & E_{23} \\
E_{31} & E_{32} & E_{33}
\end{array}\right)
\end{array}\right.
$$

(7) i.e. 


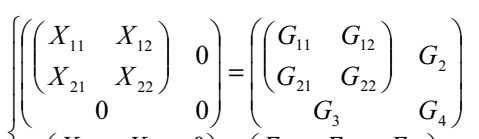

$$
\begin{aligned}
& \left\{\left(\begin{array}{ccc}
X_{33} & X_{31} & 0 \\
X_{13} & X_{11} & 0 \\
0 & 0 & 0
\end{array}\right)=\left(\begin{array}{lll}
E_{11} & E_{12} & E_{13} \\
E_{21} & E_{22} & E_{23} \\
E_{31} & F_{32} & E_{33}
\end{array}\right)\right.
\end{aligned}
$$

This proof is completed.

To sum up the above results, we obtain the detailed steps of solving (1):

(i) Let $L=\left[\begin{array}{ccc}B_{1} & B_{2} & I_{l} \\ I_{k} & I_{q} & 0\end{array}\right]$, we apply a sequence of elementary row operations on the first $l$ rows of $G$ and apply a sequence of elementary column operations on the first $k$ columns of $L$ and obtain the following form

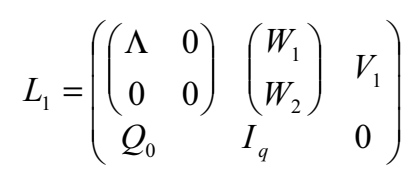

Where, $\Lambda$ is a non-degenerate upper (or lower) triangular matrix. Then, we apply a sequence of elementary row operations on the first $s$ rows of $L_{1}$ and apply a sequence of elementary column operations on the next $q$ columns of $L_{1}$ again till we obtain the following form

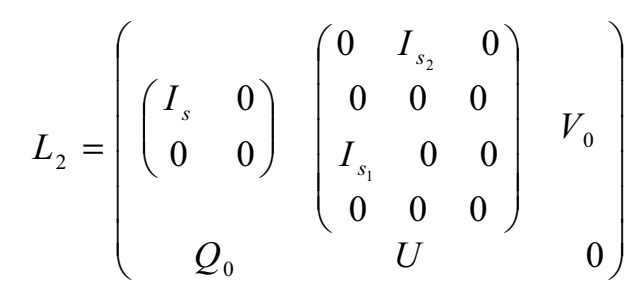

(ii) Let $M=\left(\begin{array}{cc}A_{1} & I_{m} \\ A_{2} & I_{p} \\ I_{n} & 0\end{array}\right)$, we apply a sequence of elementary row operations on the first $m$ rows of $M$ and apply a sequence of elementary column operations of the first $n$ columns of $M$ and obtain

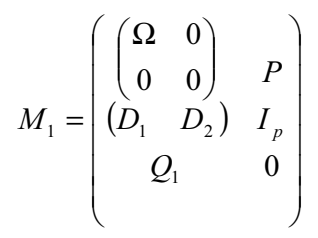

Where, $\Omega$ is a non-degenerate upper (or lower) triangular matrix. Then, we apply a sequence of elementary column operations on the first $r$ columns of $M_{1}$ and apply a sequence of elementary row operations on the next $p$ rows of $M_{1}$ again till we obtain the following form

$M_{2}=\left(\begin{array}{cccc}\left(\begin{array}{cc}I_{s} & 0 \\ 0 & 0\end{array}\right) & & P \\ \left(\begin{array}{cccc}0 & 0 & I_{s_{1}} & 0 \\ I_{s_{2}} & 0 & 0 & 0 \\ 0 & 0 & 0 & 0\end{array}\right) & V \\ & Q & & 0\end{array}\right)$

(iii) By theorem 2.2, we can discuss the all solution circumstances of (1) and obtain an expression of general solutions if it has solution. 


\section{References}

Xue You-cai, A matrix equation $A X B=C Y D$ over an arbitrary skew field, J. of Engineering Math. 15:1(1998), 139-142.

Xie Bang-jie, Self-conjugate matrices and determinant over the quarternion field, J. Natural Science of Jilin University, 2(1980), 19-35.

Xie Bang-jie, Matrices and two types of generalized Jordan's form over a ring and a skew field, J. Natural Science of Jilin University, 1(1978), 21-46.

Tu Bexun, Generalized inverses of matrices over a p-skew field, Acta. Math.Sinica, 29:2(1986), 246-248.

T.W.Hungerford, Algebra, Spring-verlag, New York, in 1980.30:5(1987), 688-694. 